\title{
Molecular Dynamics Study of Cluster Structure and Properties of Rotational Waves in Solid Nanostructures
}

\author{
I. F. Golovnev ${ }^{1, \text { a) }}$, E. I. Golovneva, ${ }^{1, \text { b) }}$, L. A. Merzhievsky ${ }^{2}$, \\ V. M. Fomin ${ }^{1}$, and V. E. Panin ${ }^{3,4}$ \\ ${ }^{1}$ Khristianovich Institute of Theoretical and Applied Mechanics SB RAS, Novosibirsk, 630090, Russia \\ ${ }^{2}$ Lavrentiev Institute of Hydrodynamics SB RAS, Novosibirsk, 630090, Russia \\ ${ }^{3}$ Institute of Strength Physics and Materials Science SB RAS, Tomsk, 634055, Russia \\ ${ }^{4}$ National Research Tomsk Polytechnic University, Tomsk, 634050, Russia \\ a) Corresponding author: golovnev@itam.nsc.ru \\ b) elena@itam.nsc.ru
}

\begin{abstract}
The paper reports a molecular dynamics analysis of rotary properties of a transformational wave generated due to compressive influence. Studies are performed in the time interval prior to the onset of elastic precursor reflection from the free boundary. It is shown that the leading front of a rotary wave coincides with the transformational wave front. The rotary wave velocity for copper is determined, being equal to $1300 \mathrm{~m} / \mathrm{s}$. The values of angular moment projections onto the coordinate axes in a plane perpendicular to wave propagation are found to be symmetrical, and their total sum equals zero.
\end{abstract}

Keywords: nanostructure, surface, fracture, rotating wave, molecular dynamics modeling

\section{INTRODUCTION}

In the paper [1], the microstructural processes in a nanocrystal of copper under the influence of the applied stress pulse have been investigated, using the molecular dynamics method. It is shown that there is a critical flow of energy, under which the defects of deformation arise in a nanocrystal as a phenomenon of self-organization of the structure in a highly non-equilibrium system. Knowing that a nanocrystal is exactly such a kind of system with a near-zero Gibbs thermodynamic potential [2], there is a wide range of transformational structural defects, qualitatively different from the dislocations in the thermodynamically stable crystals. One of these important structural defects in nanocrystals are clusters of different atomic configurations [3]. However, this issue for nanocrystalline structures has not been sufficiently investigated.

The analytical theory of nonlinear waves of structural transformations has been developed in several works [4-6]. According to one of them, the control parameter of nonlinear waves in local structural transformations is a local curvature in a highly non-equilibrium structure. In other words, the propagation of nonlinear waves of localized plastic flow and non-dislocational nature is a translation-rotation process. An experimental verification [7] of this provision under the uniaxial tension of flat samples made of metallic materials with nanostructured surface layers confirmed the prediction of the nonlinear wave theory.

In this study, the goal is the development of the research [1] in order to identify the occurrence of the cluster structure and rotational field in the propagation of nonlinear wave flow of local structural transformations in a single crystal of copper under the influence of external loads.

For this purpose the molecular dynamics method was used, and so the development and the progress of the waves generated by the external permanent compressive load $\sigma_{0}$, which operates during the time interval $T_{\sigma}$, were calculated for the nanostructure, which has the shape of a rectangular parallelepiped. At the same time, the related

International Conference on Physical Mesomechanics of Multilevel Systems 2014

AIP Conf. Proc. 1623, 171-174 (2014); doi: 10.1063/1.4898910

(C) 2014 AIP Publishing LLC 978-0-7354-1260-6/ $\$ 30.00$ 
processes in the structure at the micro level were investigated. The same physical system was used to study energy dissipation in the previous research [1]. Impulse duration $T_{\sigma}$ is also an external controllable parameter, same as the value of the external stress $\sigma_{0}$. The dimensions of the system are such that the elastic wave reaches the right side of the free boundary in $6 \mathrm{ps}$. In order to reflect the effect of the free boundary and avoid the complication of the picture of the processes at this stage, the study of all events is limited to time interval $T_{\sigma}$ equal to 6 ps. The detailed description of the physical system and the mesoanalysis of the processes are given in [1].

\section{CALCULATIONS AND RESULTS}

First let us introduce the explanation of some terms which will be used in the paper below. The term "macro" is applied to something related to the total system. The term "micro" means that the properties of an atom are referred to. And, at last, the term "meso" is used in the paper in cases when the characteristics and processes are examined in such subsystems as the atomic plane, surface atoms, etc.

For this purpose, the study of rotational properties of the wave was conducted that included a nanostructure of a mesovolume cuboid. Its length was $5 a$ ( $a$ - crystal cell size) with a square with sides equal $a / 2$ at the base. To calculate the angular momentum of the atoms along these axes, these mesovolumes were arranged so that their sides were parallel to these axes. This allowed for the calculation of the spatial distribution of the angular momentum component in relation to the axis parallel to the large generators and passing through the centers of mesovolumes' square sections.

In this paper, the widely known Verlet's numerical scheme of the second order of accuracy was used. The time step was equal to $10^{-16} \mathrm{~s}$. After disconnecting the external stress at temporal moment $T_{\sigma}$, the system becomes closed and isolated. From this point, the integration error calculation was performed:

$$
\text { err }=\frac{E_{t}-E_{T \sigma}}{E_{T \sigma}} \cdot 100 \% .
$$

Here $E_{t}$ and $E_{T \sigma}$ stand for the energy system at the time of $t$ and $T_{\sigma}$, respectively. As a result, the error in energy for the interval of $50 \mathrm{ps}$ (500000 steps of time) should not exceed $10^{-5} \%$.

Figure 1 shows the crystal in the $X Z$ plane at the time of 6 ps (black circles) and the same cooling system using the method of artificial viscosity (gray circles). To facilitate visual analysis, the scales on axes are unequal.

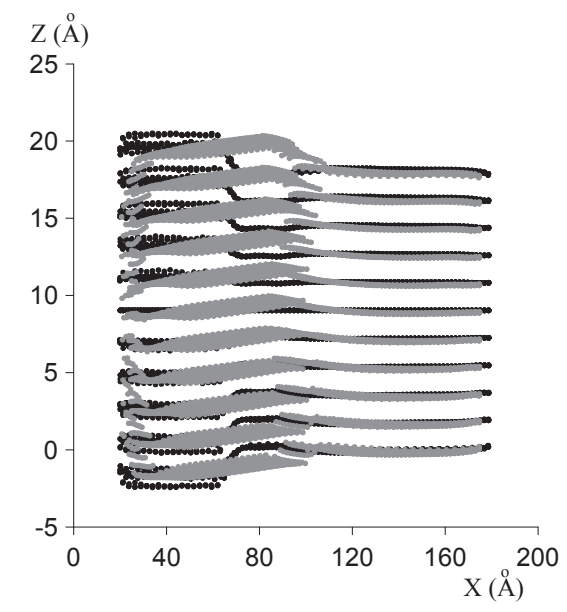

FIGURE 1. Position of atoms in the structure of the $X Z$ plane. At the time of $6 \mathrm{ps}$, the external stress is $3.6 \mathrm{GPa}$. Black circles mark the structure in the state of dynamic motion; gray circles mark it after cooling

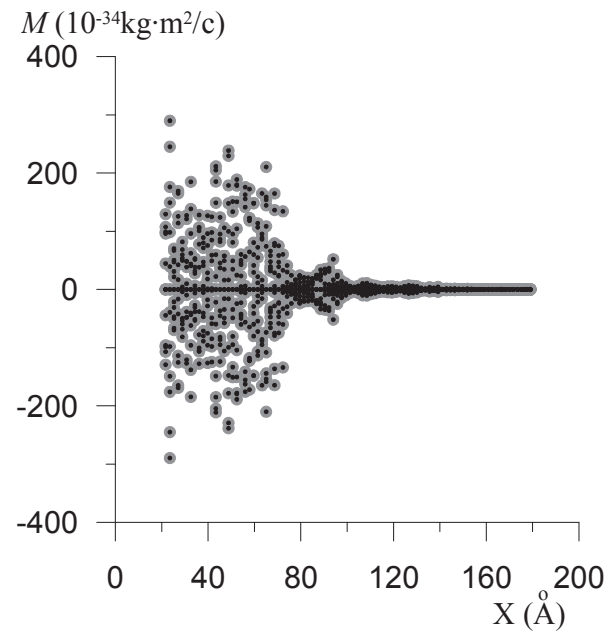

FIGURE 2. Distribution of rotation moments in mesocells of the crystal along axis $X$. Gray circles $-M_{Z}$ moments,

black circles $-M_{Y}$ moments. Time moment $t=6 \mathrm{ps,}$ $\sigma=4 \mathrm{GPa}, T_{\sigma}=6 \mathrm{ps}$ (time when the external stress was off) 


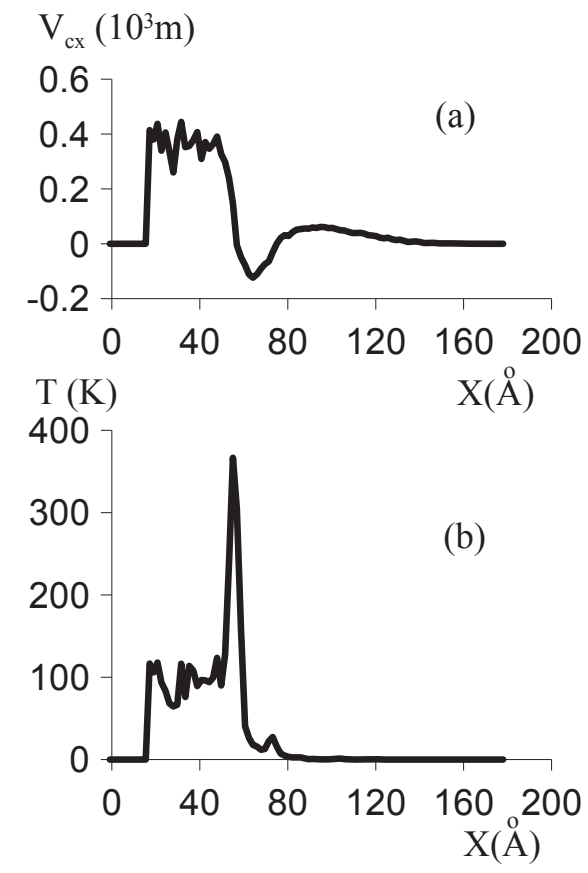

FIGURE 3. State of the crystal at time $t=5 \mathrm{ps}, \sigma=4 \mathrm{GPa}, T_{\sigma}=6 \mathrm{ps}$ : (a) velocity distribution $V_{c x}$ in the mass centers of mesocells along the axis $X$; (b) temperature distribution in mesocells along axis $X$

As it can be seen, there is a wave propagating through the crystal, in the front of which there is a significant fracture of the ideal structure. Moreover, the resulting defects can not be attributed to dislocations, they are more complicated.

It can be seen that the front edge of the wave is a transformational complex formed of multiple interfaces with different radii of curvature. Within the framework of the synergetic approach, this can be interpreted as follows.

As a result of the self-organization process, the nanostructure generates multiple interfaces in order to absorb the increasing energy flow; these interfaces are the generators of defects, which absorb much more external energy as compared to the case of the propagation of elastic waves.

This was followed by the analysis of the angular momentum in the mesovolumes mentioned above. First of all, it was necessary to determine their relative value moments, $M_{Y}^{c}, M_{Z}^{c}$, in perpendicular planes. Figure 2 shows the distribution of points along axis $X$.

It can be seen that the values of the $M_{Y}^{c}, M_{Z}^{c}$ moments in the $X$-mesovolumes are the same. Furthermore, it should be noted that the front edge of the transformational wave coincides with the front of a considerable rotational motion. In this regard, the term "rotating wave" will be used hereinafter. Additional studies of the distribution of the angular momentum in the plane perpendicular to axis $X$ have shown that their values are the same, and the sum

$$
\sum_{c} M_{Y}^{c}=0, \sum_{c} M_{Z}^{c}=0
$$

for each $X$-mesovolume equals zero. In connection with this, the illustration for the $M_{Z}^{c}$ moments will be provided below. It should be noted that the magnitude of the $M_{X}^{c}$ moments is significantly lower than that of the $M_{Y}^{c}, M_{Z}^{c}$ moments.

To determine the rotational velocity of wave propagation, the most convenient characteristics are the temperature distribution and the velocity of the mass centers of atoms in mesocells along axis $X$ in the nanostructure.

Figure 3 below shows the distribution in these time moments at the time of 5 ps. Knowing the coordinate of a certain point of the wave at certain times, it is possible to determine the speed of the transformational wave propagation under the influence of external stress. 
The average wave velocity $\left\langle V_{T}\right\rangle$, which was calculated from the peak temperature in the interval from 1 to 6 ps is $1.3 \mathrm{~km} / \mathrm{s}$. Besides determining the velocity, the conducted mesoanalysis allows us to explore the relaxation period of the rotating wave formation. A plateau with the width of $40 \AA$ and a linear increase in the velocity of the substance was formed up to 2 ps. Next, at a distance of about $10 \AA$, the velocity increased by approximately 4 times. Simultaneously, the peak temperature of up to $50 \mathrm{~K}$ is formed over the plateau. Furthermore, until 4 ps the plateau of mass velocity became an S-shaped structure with a negative velocity. Simultaneously, the temperature wave was formed with a sharp peak at $300 \mathrm{~K}$.

\section{CONCLUSION}

Within the framework of the molecular dynamics method in the mechanical behavior at the micro level of copper nanocrystal, the following results have been obtained:

1. The formation of a rotating wave, in which there is an increased generation of cluster defects with various atomic configurations, has been identified.

2. It has been shown that the front edge of a rotating wave is a complex of formed multiple interfaces with different radii of curvature.

3. It has been shown that the nanostructure, as a result of a self-organization process, generates multiple interfaces with different radii of curvature that generate metastable defects and lower the thermodynamic disequilibrium of a nanocrystal;

4. The rotational velocity of the front wave, which is about $1300 \mathrm{~m} / \mathrm{s}$, has been calculated.

5. It has been demonstrated that the front of the rotation wave is about $70 \AA(7 \mathrm{~nm})$ and has a complicated structure. At the front edge of the front, the mass velocity is negative (the substance moves against the motion of the wave, which is associated with the formation of the cluster structure). Then the velocity becomes positive (400 m/s) and, in its amplitude, exceeds the velocity at the front edge by about 2 times $(-200 \mathrm{~m} / \mathrm{s})$. Above the front with a negative velocity, the negative peak of temperature is formed, where the temperature $(400 \mathrm{~K})$ is about 4 times higher than that at the front $(100 \mathrm{~K})$.

\section{ACKNOWLEDGEMENTS}

This work was funded by the interdisciplinary integration project of SB RAS No. 78.

\section{REFERENCES}

1. I. F. Golovnev, E. I. Golovneva, L. A. Merzhievsky, and V. M. Fomin, Phys. Mesomech. 16(4), 294 (2013).

2. V. E. Panin and V. E. Egorushkin, Phys. Mesomech. 12(5-6), 204 (2009).

3. E. Infeld and G. Rowlands, Nonlinear Waves, Solitons and Chaos (Cambridge University Press (Verlag), Cambridge, 2000).

4. V. E. Panin, V. E. Egorushkin, and A. V. Panin, Phys. Mesomech. 9(3-4), 9 (2006).

5. D. J. Korteveg and F. de Vries, Phil. Mag. 39, 422 (1985).

6. V. E. Egorushkin, Russ. Phys. J. 35(4), 316 (1992).

7. V. E. Panin, V. E. Egorushkin, and A. V. Panin, Physics-Uspekhi 55(12), 1351 (2012). 